\title{
Melatonin for sleep problems in children with neurodevelopmental disorders: randomised double masked placebo controlled trial
}

In this Research paper by Paul Gringras and colleagues (BMJ 2012;345:e6664, doi:10.1136/bmj.e6664), the full list of members of the MENDS Study Group was omitted. In addition to the named authors the group comprises:

Fiona Atkin, Lucy Bray (research nurses, Royal Liverpool Children's Hospital), Louise Barnes, Michelle Cooper, Jasmine Heslop, Su Wilkins (research nurses, Royal Devon and Exeter Hospital, Torbay Hospital), Emily Benson, Jacqui Dalglish, Lisa Hydes, Julie Menzies (research nurses, Birmingham Children's Hospital), Jackie Bradley, Heather Rostron (research nurses, Blackpool Victoria Hospital), Thomas Bourgeron (genetics analyses, Pasteur Institute, Paris), Helen Clark, Sheila Fox, Vicky Payne, Claire Roberts, Heather Smee, Catherine Upton (research nurses, Southmead Hospital, Bristol), Christina Daines, Rachel Voce (research nurses, Leicester Royal Infirmary), Sarah Dyas, Teresa Moorcroft (research nurses, Arrowe Park Hospital), Penny Erskine, Penny Scardifield (research nurses, Nottingham City Hospital), Melanie Farman (research nurse, Royal Manchester Children's Hospital), Fabien Fauchereau (statistical analyses of genetic data), Jayaprakash Gosalakkal (principal investigator, Leicester Royal Infirmary), Hany Goubran Botros (generation of genetic data and their sequenced analyses), Val Harpin (principal investigator, Sheffield Children's Hospital), Cathy Hill (principal investigator, Southampton General Hospital), Lorraine Hodson (research nurse, Evelina Children's Hospital, London), Simone Holley, Cilla Long, Emma Macleod (research nurses, Southampton General Hospital), Anwen Howells, Alison McQueen, Ann Russell (research nurses, Children's Hospital for Wales), Adrian Hughes (principal investigator, Arrowe Park Hospital), Guillaume Huguet (generation of genetic data and statistical analyses), Xenya Kantaris, Louise Spencer Walsh (research nurses, University College London Hospitals), Natalie Lemière (generation of candidate gene sequences), Ros Loxton (research nurse, Queen Mary's Hospital), Samantha Jones, Joanna Smith, Vanessa Unsworth, Lynda Viles (research nurses, Derbyshire Children's Hospital, Chesterfield Royal Hospital, Nottingham City Hospital), Andrea Jorgensen (co-writing of protocol amendment and statistical analysis plan for genetic substudy, advice regarding analysis of genetic data, and integration of genetic results into report), Mark Lathrop (generation of high throughput genetic data), Tim Martland (principal investigator, Royal Manchester Children's Hospital), Andrew McKay (statistical analyses of secondary outcomes, Liverpool), Benita Middleton (advice and facilitation of salivary melatonin measurements, University of Guildford, Surrey), Tracey Oakden (research nurse, University College London Hospitals), Cécile Pagan (generation of genetic data and statistical analyses), Karen Pratt (research nurse, Evelina Children's Hospital, London), Philip Preece (principal investigator, Chesterfield Royal Hospital), Béatrice Regnault (generation of high throughput genetic data), Clive Sainsbury (principal investigator, Torbay Hospital), Debra Skene (advice and facilitation of salivary melatonin measurements, professor, University of Guildford, Surrey), Coral Smith (research nurse, Derbyshire Children's Hospital), Ron Smith (principal investigator, Royal Devon and Exeter Hospital), Masako Sparrowhawk (research nurse, John Radcliffe Hospital, Oxford), Louise Strickland (research nurse, Royal Devon and Exeter Hospital, Torbay Hospital), Jacqui Tahari, Helen Wilde (research nurses, Royal Liverpool Children's Hospital (Alder Hey), Arrowe Park Hospital), Johann te Water Naude (principal investigator, Children's Hospital for Wales), Amanda Thobinson (research nurse, Royal Liverpool Children's Hospital, Royal Manchester Children's Hospital), Megan Thomas (principal investigator, Blackpool Victoria Hospital), Jeremy Turk (principal investigator, Queen Mary's Hospital), Evangeline Wassmer (principal investigator, Birmingham Children's Hospital), Jacqui Woods (research nurse, Royal Manchester Children's Hospital, Blackpool Victoria Hospital), Zenobia Zaiwalla (principal investigator, John Radcliffe Hospital, Oxford), Diana Zelenica (generation of high throughput genetic data).

Cite this as: BMJ 2012;345:e7618

๑ BMJ Publishing Group Ltd 2012 